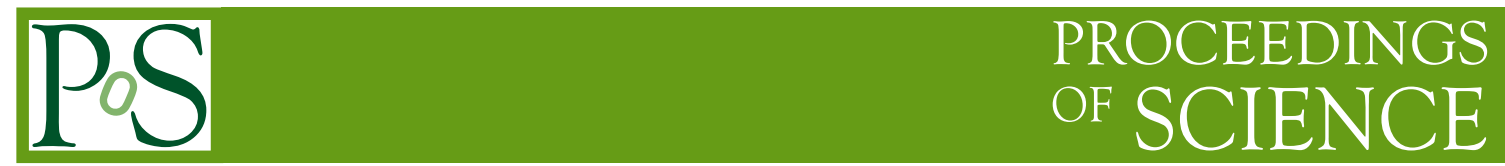

\title{
Flavor Questions for the LHC
}

\author{
Jonathan Rosner* \\ Enrico Fermi Institute and Department of Physics \\ University of Chicago \\ E-mail: rosner@hep.uchicago.edu
}

The physics underlying quark and lepton masses and mixings (the "flavor problem") is the least well understood aspect of the Standard Model. Some questions of flavor physics, and ways in which the LHC can help shed light on this problem, are described.

Flavor Physics and CP Violation 2009

May 27-June 12009

Lake Placid, NY, USA

* Speaker. 


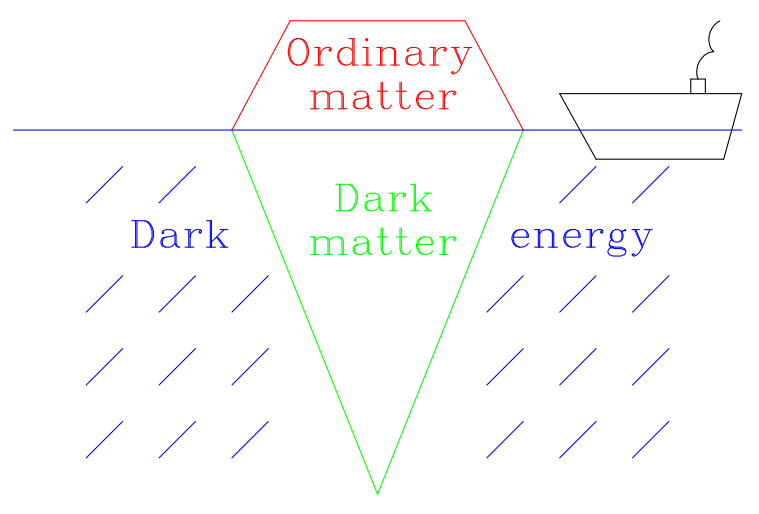

Figure 1: Illustration of our relation to ordinary matter.

\section{Introduction}

Flavor is the least well understood aspect of the Standard Model. Ordinary matter makes up $4.6 \%$ of the known energy density of Universe, while dark matter comprises another $23 \%$ [1]]. We have little clue as to its nature. Dark energy accounts for the remaining $72 \%$; we know even less about it. Ordinary quarks and leptons thus represent just the tip of a very big iceberg (see Fig. 1). We need to understand the rest of the iceberg (and the sea in which it swims) in order to understand the pattern underlying the known forms of matter.

\section{Quark patterns}

We are accumulating very precise information about the pattern of quark masses and couplings. If we regard the weak charge-changing transitions $u \leftrightarrow d, c \leftrightarrow s$, and $t \leftrightarrow b$ as of relative strength $\mathscr{O}(1)$, then the transitions $u \leftrightarrow s$ and $c \leftrightarrow d$ are of order $\lambda \sim 0.23$; the transitions $c \leftrightarrow b$ and $t \leftrightarrow s$ are of order $\lambda^{2} \sim 0.04$, and the transitions $u \leftrightarrow b$ and $t \leftrightarrow d$ are of order $\lambda^{3} \sim 0.01$ or less. This information is encoded in the unitary Cabibbo-Kobayashi-Maskawa (CKM) matrix [2, 3], whose invariant phase describes CP violation. The CKM matrix arises from the same (unknown) physics giving rise to the pattern of quark masses. A related pattern arises for the leptons, which differ by having very small neutrino masses and large mixings.

What kind of physics is giving rise to this pattern? It is likely we will understand it much more fully if we know how much of the pattern we are already seeing. Two familiar examples, illustrated in Fig. 2, give conflicting prospects for understanding the flavor problem.

In the periodic table of the elements, the variations of the pattern are the key to its comprehension. Each element has a different nuclear charge; the electron shell structure governs chemistry. Through this pattern, the existence of the element Technetium was predicted.

The orbits of the planets (out to Uranus) obey the approximate relation (Titius/Bode law) $a(\mathrm{AU})=0.4+0.3 k$, where $k=0,1,2,4,8$. This rule predicted the orbits of the large asteroid Ceres and the planet Uranus. However, it failed to predict the orbit of Neptune. Pluto is approximately where Neptune should have been; other dwarf planets don't fit; and there is no dynamical explana- 

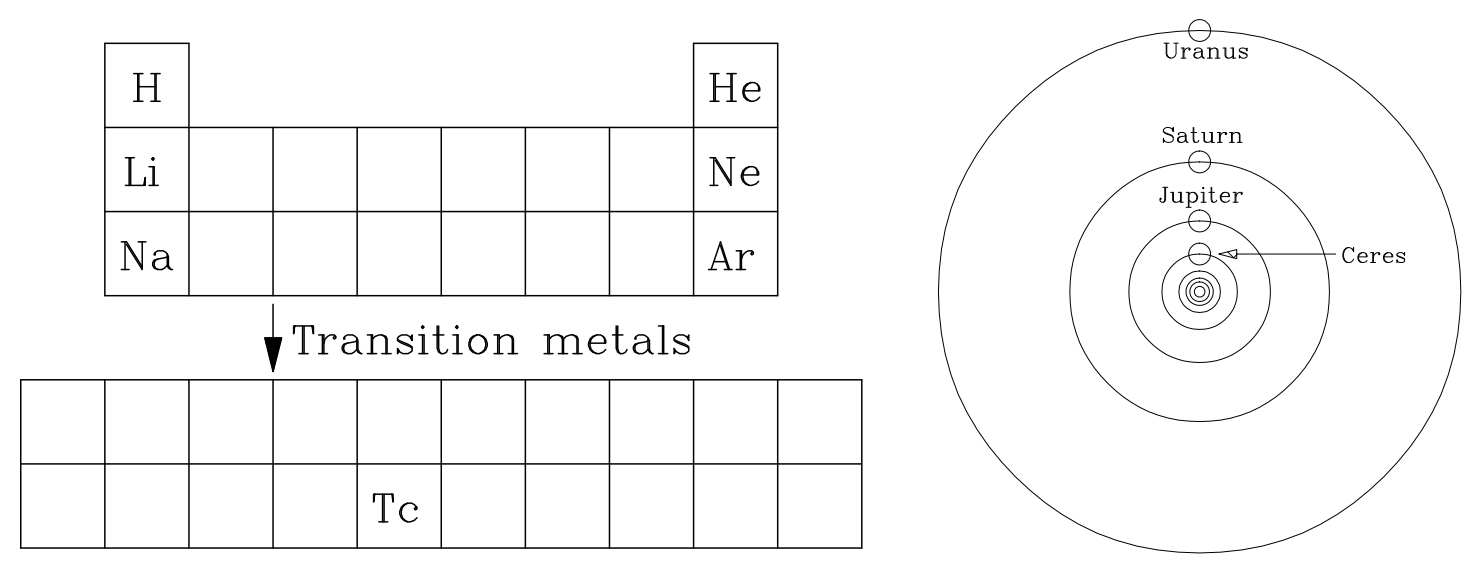

Figure 2: Patterns of the elements (left) and planets (right).

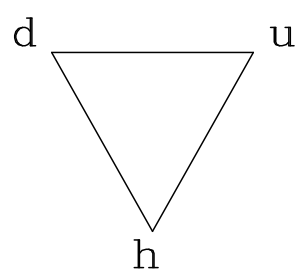

$(3,1,3)$

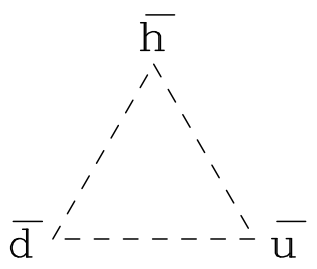

$\left(1,3^{*}, 3^{*}\right)$

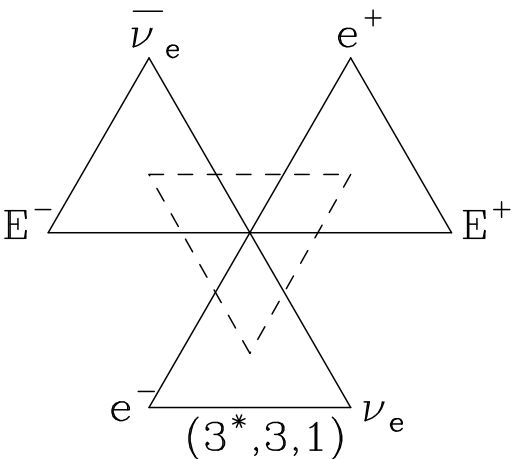

Figure 3: Members of the 27-plet of $\mathrm{E}_{6}$, depicted in terms of their quantum numbers in $\mathrm{SU}(3)_{\mathrm{L}}$ (solid triangles) and $\mathrm{SU}(3)_{\mathrm{R}}$ (dashed triangles). From left to right: quarks, antiquarks, and leptons.

tion for the rule. Simulations can give similar relations, in analogy to "anarchy" [4] in models of quark and lepton masses.

Will the pattern of quark and lepton masses reflect some underlying structure, as in the periodic table, or essential anarchy, as in planetary orbits? We are likely to know much more once the nature of dark matter is revealed.

Examples of extensions of the Standard Model include a fourth family of quarks and leptons, extended grand unified theories (GUTs), and Kaluza-Klein excitations. To take the example of GUTs, in SU(5) the representations $5^{*}+10$ account for all known left-handed quarks and leptons in a family, while these are combined into one 16-dimensional spinor of $\mathrm{SO}(10)$ with the addition of a left-handed antineutrino (presumably with large Majorana mass). In the exceptional group $\mathrm{E}_{6}$ which contains $\mathrm{SO}(10)$ as a subgroup, the fundamental 27-dimensional representation involves adding an $\mathrm{SO}(10)$ 10-plet and singlet to the known 16-plet. $\mathrm{E}_{6}$ has a subgroup $\mathrm{SU}(3)_{\mathrm{L}} \otimes \mathrm{SU}(3)_{\mathrm{R}} \otimes$

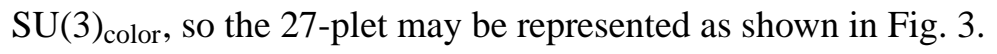

The new fermions consist of isosinglet $Q=-1 / 3$ quarks $h$; vector-like leptons $E^{ \pm}$and their neutrinos $v_{E}, \bar{v}_{E}$ (center of right-hand figure); and a new sterile neutrino $n$ (center of right-hand 


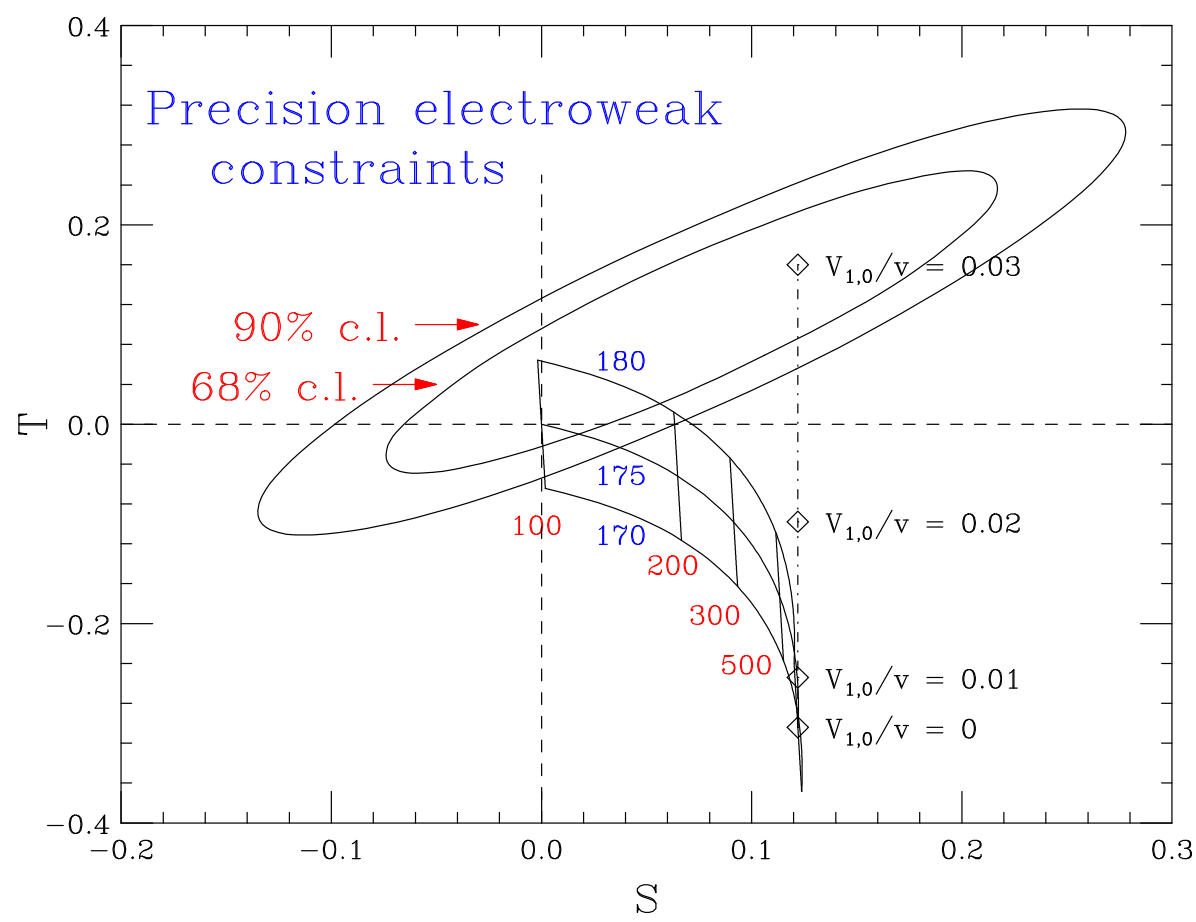

Figure 4: $S-T$ plot based on precision electroweak constraints (see text).

figure). The $h$ could mix with $b$ and be responsible for $m_{b} \ll m_{t}$ [5]. Searches at Fermilab exclude masses up to $\sim 300 \mathrm{GeV}$ [6].

If a fourth quark-lepton family exists [7], its neutrino must be heavier than $\sim M_{Z} / 2$, as the invisible width of the $Z$ indicates that only three neutrinos are light [8]. New particles in loops (such as fourth-family members) will affect $W, Z, \gamma$ propagators and SM coupling relations. These effects may be described by parameters $S$ and $T$ [9]:

$$
\frac{G_{F}}{\sqrt{2}}=\left(1+\frac{\alpha S}{4 \sin ^{2} \theta}\right) \frac{g^{2}}{8 M_{W}^{2}}, \quad \frac{G_{F} \rho}{\sqrt{2}}=\frac{g^{2}+g^{\prime 2}}{8 M_{Z}^{2}}, \quad \rho \equiv 1+\alpha T, \quad \alpha \simeq 1 / 129 .
$$

Each new quark-lepton family contributes $\Delta S=2 /(3 \pi) \simeq 0.2, \Delta T \simeq 0.4\left(m_{t^{\prime}}^{2}-m_{b^{\prime}}^{2}\right) /(100 \mathrm{GeV})^{2}$. The latter contribution is particularly important. In Fig. Đwe plot the allowed region of $S$ and $T$ based on precision electroweak constraints [10]. Also shown are predictions of the Standard Model for Higgs boson masses of 100-500 GeV (nearly vertical contours) and top quark masses of 170 , 175 , and $180 \mathrm{GeV}$ (curved contours). The vertical dot-dashed line shows the effect of a small triplet-Higgs VEV $V_{1,0}$ (up to 0.03 of the Standard Model VEV $v=246 \mathrm{GeV}$ ), where the subscripts denote weak isospin and weak hypercharge. The triplet Higgs leads to $\Delta \rho=4\left(V_{1,0} / v\right)^{2}$. A large $t^{\prime}-b^{\prime}$ mass splitting behaves like a triplet Higgs, causing positive $\Delta \rho=\alpha \Delta T$ and allowing the relaxation of the usual stringent upper limit on the Higgs boson mass [11]. 


\section{CKM matrix parameters}

In the parametrization suggested by Wolfenstein [12],

$$
V=\left[\begin{array}{lll}
V_{u d} & V_{u s} & V_{u b} \\
V_{c d} & V_{c s} & V_{c b} \\
V_{t d} & V_{t s} & V_{t b}
\end{array}\right] \simeq\left[\begin{array}{ccc}
1-\frac{\lambda^{2}}{2} & \lambda & A \lambda^{3}(\rho-i \eta) \\
-\lambda & 1-\frac{\lambda^{2}}{2} & A \lambda^{2} \\
A \lambda^{3}(1-\rho-i \eta) & -A \lambda^{2} & 1
\end{array}\right]
$$

the parameters are known fairly accurately: $\lambda \simeq 0.2255, A \simeq 0.81,0.14 \leq \rho \leq 0.18$, and $0.34 \leq$ $\eta \leq 0.36$. (Two groups [13, 14] obtain slightly different parameters when fitting observables.) The unitarity of $V$ implies (e.g.) $V_{u d} V_{u b}^{*}+V_{c d} V_{c b}^{*}+V_{t d} V_{t b}^{*}=0$ or dividing by the middle term, $(\rho+i \eta)+(1-\rho-i \eta)=1$. This generates the unitarity triangle (UT), whose angles opposite the sides $1, \rho+i \eta, 1-\rho-i \eta$ are, respectively, $\alpha, \beta$, and $\gamma$. One learns its shape from such observables as kaon $\mathrm{CP}$ violation (essentially constraining $\eta(1-\rho)$ ), $B-\bar{B}$ mixing (constraining $|1-\rho-i \eta|$, given suitable hadronic information), and charmless $B$ decays (constraining $|\rho+i \eta|$ ). Direct measurements of angles satisfy $\alpha+\beta+\gamma=\pi$ [15], with

$$
\alpha=\left(89.0_{-4.2}^{+4.4}\right)^{\circ}, \beta=(21.0 \pm 0.9)^{\circ}, \gamma=\left(70_{-29}^{+27}\right)^{\circ} \text {. }
$$

The large error on $\gamma$ highlights the importance of improving direct measurements of it, one of the goals of LHCb. Measurements of sides of the UT are more constraining, as we shall now see.

\section{Mixing of strange $B$ 's}

\subsection{Constraint on CKM parameters}

A $\bar{B}_{s}^{0}=b \bar{s}$ can mix with a $B_{s}^{0}=s \bar{b}$ by means of box diagrams involving exchange of a pair of $W$ bosons and intermediate $u, c, t$ quarks. The heavy top quarks provide the dominant constribution, so $\bar{B}_{s}^{0}-B_{s}^{0}$ mixing is stronger than $\bar{B}^{0}-B^{0}$ mixing because $\left|V_{t s} / V_{t d}\right| \simeq 5$. As CKM unitarity implies $\left|V_{t s}\right| \simeq\left|V_{c b}\right| \simeq 0.041$ (and hence $\left|V_{t s}\right|$ is well-known), $B_{s}-\bar{B}_{s}$ mixing probes hadron physics. The matrix element between $B_{s}$ and $\bar{B}_{s}$ involves a combination $f_{B_{s}}^{2} B_{B_{s}}: f_{B_{s}}$ is the " $B_{s}$ decay constant" (the matrix element of $b \bar{s}$ operator between $B_{s}$ and vacuum), and $B_{B_{s}} \simeq 1$ parametrizes degree to which $W$ exchange graphs dominate mixing. A recent prediction of lattice QCD [16], $f_{B_{s}} \sqrt{B_{B_{s}}} /\left[f_{B} \sqrt{B_{B}}\right]=1.258 \pm 0.033$, when combined with the well-measured $B^{0}-\bar{B}^{0}$ mixing amplitude $\Delta m_{d}=(0.507 \pm 0.005) \mathrm{ps}^{-1}$ and the CDF $B_{s}$ mixing measurement at Fermilab [17], $\Delta m_{s}=$ $(17.77 \pm 0.10 \pm 0.07) \mathrm{ps}^{-1}$, gives $\left|V_{t d} / V_{t s}\right|=0.214 \pm 0.005$ and hence $|1-\rho-i \eta|=0.950 \pm 0.026$, implying $\gamma=(72 \pm 5)^{\circ}$. This is a great improvement over the value based on $\Delta m_{d}$, which was subject to uncertainty in $f_{B}$. The study of $B^{+} \rightarrow D^{0}\left(\bar{D}^{0}\right) K^{+}$may improve this accuracy eventually, with help from information on the strong phases in the $K_{S} \pi^{+} \pi^{-}$Dalitz plot [18].

\subsection{Mixing and $\mathrm{CP}$ violation}

In the Standard Model (SM), $B_{s} \rightarrow J / \psi \phi$ is expected in SM to have a small CP asymmetry, governed by the $B_{s}-\bar{B}_{s}$ mixing phase $\phi_{M}=-2 \beta_{s}$, where

$$
\beta_{s} \equiv \operatorname{Arg}\left(-V_{t s} V_{t b}^{*} / V_{c s} V_{c b}^{*}\right)=\lambda^{2} \eta \simeq 0.02 \text { with } \lambda=0.2255 \pm 0.0019, \eta \simeq 0.36 \text {. }
$$




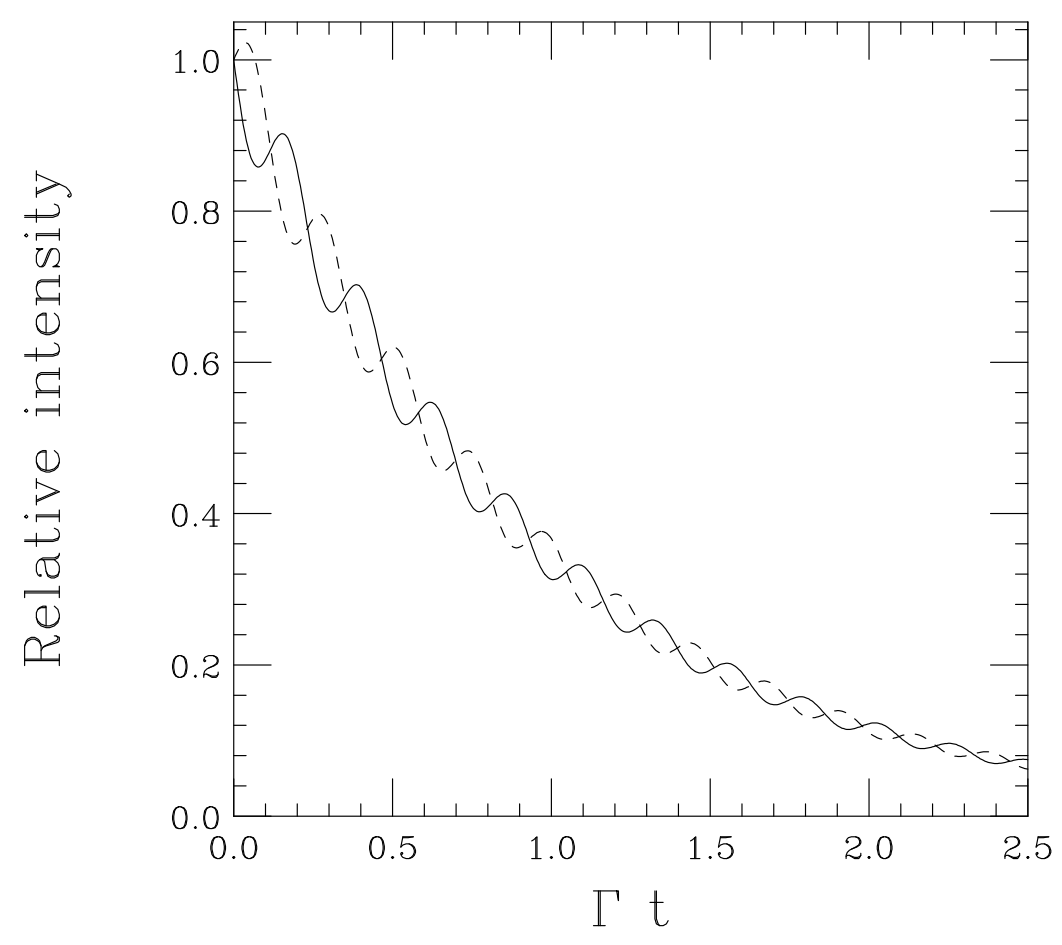

Figure 5: Assumed time-dependence of signals for functions $\mathscr{T}_{+}$with tagged initial $B_{S}$ (solid) and $\bar{B}_{S}$ (dashed). Based on best-fit parameters of Ref. [15]. Curves for $\mathscr{T}_{-}$are similar.

From angular distributions of decay products one must extract three independent partial waves $(L=0,1,2)$ or three independent amplitudes $A_{0}, A_{\|}, A_{\perp}$. At the Fermilab Tevatron, both CDF [19] and D0 [20] favor a mixing phase differing from $-2 \beta_{s}$. Defining $\phi_{B_{s}}=\beta_{s}+\phi_{M} / 2$, the HFAG average [15, 21] is $\phi_{B_{s}} \in[-163,-95]^{\circ},[-84,-17]^{\circ}, 2.2 \sigma$ away from the SM. The width difference between $\mathrm{CP}=+$ and $\mathrm{CP}=-$ mass eigenstates, $\Delta \Gamma_{s} \simeq 0.1 \mathrm{ps}^{-1}$, is compatible with SM predictions [22].

A discrete ambiguity $\phi_{M} \rightarrow \pi-\phi_{M}$ is associated with uncertainty in the strong phases $\delta_{\|} \equiv$ $\operatorname{Arg}\left(A_{\|} A_{0}^{*}\right), \delta_{\perp} \equiv \operatorname{Arg}\left(A_{\perp} A_{0}^{*}\right)$. It can be eliminated by comparison with $B^{0} \rightarrow J / \psi K^{* 0}$ as most contributions are similar [23]; the phases are predicted to be equal within $10^{\circ}$.

There are plenty of models that can accommodate a $B_{s}-\bar{B}_{s}$ mixing phase larger than in the SM. For examples ("littlest Higgs," extra dimensions, etc.) see Ref. [24].

\subsection{Time-dependences}

In Ref. [23] it was noted that the large phase claimed by CDF and D0 for $B_{s}-\bar{B}_{s}$ mixing should lead to an explicit time-dependence which exhibits $\mathrm{CP}$ violation. Observing this will not be easy, as the flavor oscillations are quite rapid (recall the large value of $\Delta m_{s}$ ). However, with $\phi_{M}=-44^{\circ}$ and $\Delta \Gamma / \Gamma=0.228$, the central values quoted in Ref. [15], the oscillations should be visible, as illustrated in Fig. 5. Here we have defined functions

$$
\left.\mathscr{T}_{ \pm} e^{-\Gamma t}\left[\cosh (\Delta \Gamma t) / 2 \mp \cos \left(\phi_{M}\right) \sinh (\Delta \Gamma t) / 2\right) \pm \eta \sin \left(\phi_{M}\right) \sin \left(\Delta m_{s} t\right)\right],
$$


associated with $\left|A_{\|}\right|^{2}$ and $\left|A_{\perp}\right|^{2}$, respectively, which may be obtained from an angular analysis of decay products. One isolates $\mathrm{CP}$ violation by tagging at $t=0: \eta= \pm 1$ for tagged $\left(B_{s}, \bar{B}_{s}\right)$.

The last term in Eq. (4.2) contains the rapid time oscillations, and changes sign with the sign $\eta$ of the tag. We have assumed the tagging parameter $\eta$ to include a dilution factor 0.11 . A plot such as Fig. 5 would be clear evidence for non-standard CP violation in $B_{s} \rightarrow J / \psi \phi$. Such oscillations would probably be too small to see in the Standard Model.

\section{5. $B \rightarrow K \pi, \pi \pi$}

Some time ago it was predicted that the $\mathrm{CP}$ asymmetries in $B^{0} \rightarrow K^{+} \pi^{-}$and $B^{+} \rightarrow K^{+} \pi^{0}$ would be equal if a color-suppressed amplitude contributing to the latter process were neglected [25]. The graphical representations of various amplitudes are shown in Fig. 6, and their contributions to $B \rightarrow K \pi$ processes are shown in Table 11 where we have included a small "annihilation" (A) contribution. The amplitudes denoted by small letters are related to those with large letters by the inclusion of electroweak penguin contributions:

$$
t \equiv T+P_{\mathrm{EW}}^{C}, c \equiv C+P_{\mathrm{EW}}, p \equiv P-(1 / 3) P_{\mathrm{EW}}^{C}
$$
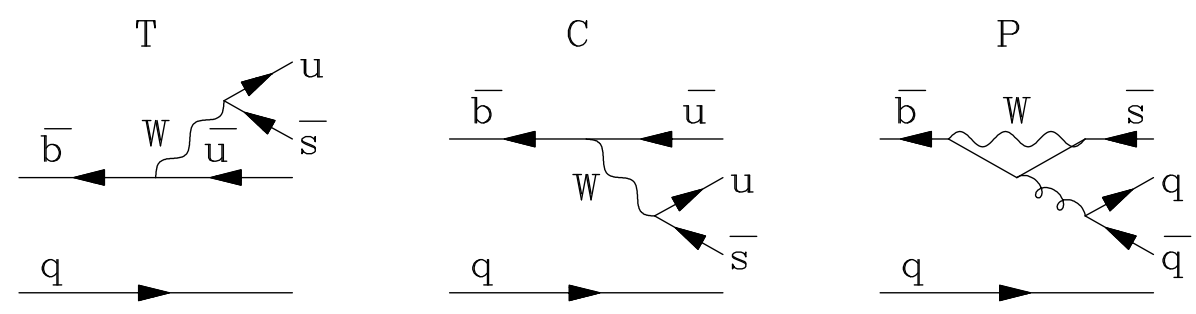

Figure 6: Amplitudes contributing to $B \rightarrow K \pi$ decay modes. $T$ : color-favored tree; $C$ : color-suppressed tree; $P$ : penguin. The annihilation graph $A$ is not shown.

Table 1: Contributions of amplitudes to $B \rightarrow K \pi$ decay modes.

\begin{tabular}{cccc}
\hline \hline Decay & Amplitude & BR $\left(10^{-6}\right)$ & $A_{C P}$ \\
\hline$B^{0} \rightarrow K^{+} \pi^{-}$ & $-(t+p)$ & $19.4 \pm 0.6$ & $-0.097 \pm 0.012$ \\
$B^{+} \rightarrow K^{+} \pi^{0}$ & $-(t+p+c+A) / \sqrt{2}$ & $12.9 \pm 0.6$ & $0.050 \pm 0.025$ \\
$B^{0} \rightarrow K^{0} \pi^{0}$ & $(p-c) / \sqrt{2}$ & $9.8 \pm 0.6$ & $0.00 \pm 0.10$ \\
$B^{+} \rightarrow K^{0} \pi^{+}$ & $p+A$ & $23.1 \pm 1.0$ & $0.009 \pm 0.025$ \\
\hline \hline
\end{tabular}

However, the color-suppressed amplitude is not negligible. An SU(3) fit to $B \rightarrow(K \pi, \pi \pi)$ [26] finds $|C / T|=0.46_{-0.30}^{+0.43}, \operatorname{Arg}(C / T)=(-119 \pm 15)^{\circ}$. These values have been confirmed in a more recent analysis [27]. They lead to a significant difference between the $\mathrm{CP}$ asymmetries in $B^{0} \rightarrow K^{+} \pi^{-}$and $B^{+} \rightarrow K^{+} \pi^{0}$. So, what's the problem? Why has this difference in CP asymmetries been repeatedly quoted as evidence for new physics? 
The debate turns on whether a priori calculations of $C$, which give a smaller-than-observed value, can be trusted. A large $C$ also is needed to understand the larger-than-expected value of $\mathscr{B}\left(B^{0} \rightarrow \pi^{0} \pi^{0}\right)=(1.55 \pm 0.19) \times 10^{-6}$. The fact that no similar enhancement of $C$ appears needed in $B \rightarrow \rho \rho$ has been ascribed in Ref. [27] to a special role for pseudoscalars. It also has been explained [28] in terms of rescattering: as $\mathscr{B}(B \rightarrow \rho \rho) \gg \mathscr{B}(B \rightarrow \pi \pi)$, the rescattering $(\rho \rho \rightarrow \pi \pi)$ is more significant than $(\pi \pi \rightarrow \rho \rho)$, implying a greater fraction of $C$ in $\pi \pi$ than in $\rho \rho$. The rescattering via $\bar{b} \rightarrow \bar{c} c \bar{s}$ also is a likely source of the enhanced $\bar{b} \rightarrow \bar{s}$ "charming" penguin.

The consistency of a unified description of $B \rightarrow K \pi \mathrm{CP}$ asymmetries may be tested by a robust sum rule for $A_{C P}$ which is satisfied as long as there are no new-physics sources of a $\Delta I=1$ amplitude [29]:

$$
\Delta\left(K^{+} \pi^{-}\right)+\Delta\left(K^{0} \pi^{+}\right)=2 \Delta\left(K^{+} \pi^{0}\right)+2 \Delta\left(K^{0} \pi^{0}\right), \Delta(f) \equiv \Gamma(\bar{B} \rightarrow \bar{f})-\Gamma(B \rightarrow f),
$$

which predicts $A_{C P}\left(B^{0} \rightarrow K^{0} \pi^{0}\right)=-0.148 \pm 0.044$, to be compared with the experimental value $-0.01 \pm 0.10$. (Furthermore, flavor SU(3) implies a large $A_{C P}\left(B^{0} \rightarrow \pi^{0} \pi^{0}\right) \simeq 0$.8.) The SM seems to be able to accommodate a modestly large value of $C$; there is no need for new-physics scenarios involving a $P_{\mathrm{EW}}$ contribution to $c=C+P_{\mathrm{EW}}$. The $A_{C P}$ sum rule provides a diagnostic for $\Delta I=1$ new physics [30]. One must measure $A_{C P}\left(B^{0} \rightarrow K^{0} \pi^{0}\right)$ to 0.03 or better.

\section{Inclusive $D \rightarrow \omega X$}

CLEO's measurement of a large inclusive branching fraction $\mathscr{B}\left(D_{s}^{+} \rightarrow \omega X\right)=(6.1 \pm 1.4) \%$ [31] was a surprise. Before this measurement, the only known $D_{s}$ mode involving $\omega$ was $D_{s}^{+} \rightarrow$ $\pi^{+} \omega$ with branching fraction $\mathscr{B}=(0.25 \pm 0.09 \%)$ [32]. Now, however, CLEO has discovered a number of other $D_{s}$ exclusive modes involving $\eta$ [33], accounting for a total of $(5.4 \pm 1.0) \%$ of $D_{s}$ decays.

Mechanisms for $D_{s}^{+} \rightarrow \omega X^{+}$are not so obvious: one often has to get rid of an $s \bar{s}$ pair. Two candidate subprocesses are shown in Fig. ฤ.
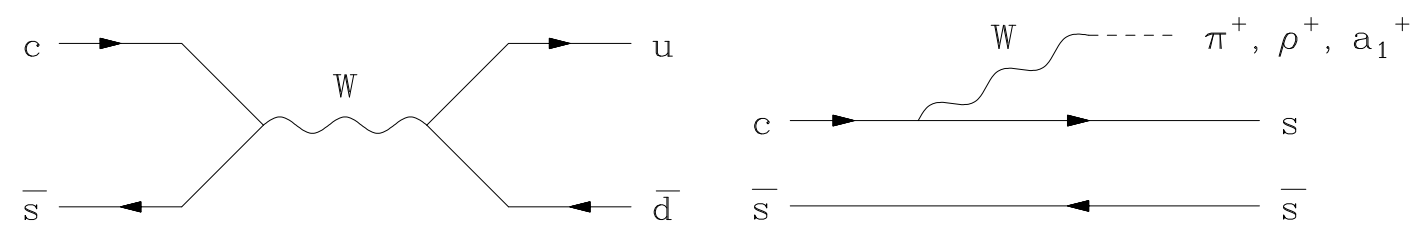

Figure 7: Diagrams contributing to $D_{s} \rightarrow \omega X$. Left: annihilation; right: color-favored tree.

In the left-hand diagram, the process $D_{s}^{+} \rightarrow\left(\right.$ virtual $\left.W^{+}\right) \rightarrow u \bar{d}$ is helicity-suppressed, and G-parity forbids production of the final states $\pi^{+} \omega$ and $(3 \pi)^{+} \omega$. In the right-hand diagram, the subprocess $c \rightarrow u \bar{d} s$ with a spectator $s$ could give $\omega \pi^{+} \eta\left[34\right.$. One also could get $\omega\left(\pi^{+}, \rho^{+}, a_{1}^{+}\right)$if the transition $s \bar{s} \rightarrow \omega$ is somehow not subject to the usual Okubo-Zweig-Iizuka (OZI) suppression [35]. In that case one might expect $D_{s} \rightarrow \omega \ell^{+} v_{\ell}$ to be observable. Helicity suppression also seems not to be apparent in CLEO's result [36] $\mathscr{B}\left(D_{s} \rightarrow p \bar{n}\right)=\left(1.30 \pm 0.36_{-0.16}^{+0.12}\right) \times 10^{-3}$, given a reasonable form factor for the weak current to produce $p \bar{n}$. 


\section{Some models for new physics}

Extra $Z$ bosons arise in many extensions of the SM. They are not guaranteed to have flavordiagonal couplings if SM fermions also mix with new fermions in such extensions. For example, GUTs based on the exceptional group $\mathrm{E}_{6}$ have two extra $Z$ bosons $Z_{\chi}, Z_{\psi}$ (only one linear combination of which may be relatively light) and extra isoscalar quarks with $Q=-1 / 3$ which can mix with $d, s, b$.

Many grand unified theories have a $\mathrm{SU}(4)_{\text {color }} \times \mathrm{SU}(2)_{\mathrm{L}} \times \mathrm{SU}(2)_{\mathrm{R}}$ subgroup [37]. $\mathrm{SU}(4)_{\text {color }}$ unifies quarks and leptons and contains $\mathrm{U}(1)_{\mathrm{B}-\mathrm{L}}$ and leptoquarks; $\mathrm{SU}(2)_{\mathrm{R}}$ has right-handed $W$ 's and a $\mathrm{U}(1)_{\mathrm{R}}$ such that the electromagnetic charge is $Q=I_{3 \mathrm{~L}}+I_{3 \mathrm{R}}+(B-L) / 2$. Leptoquarks can contribute to leptonic meson decays; right-handed $W$ 's contribute to mixing; there are strong constraints on $W_{L}-W_{R}$ box diagrams (see, e.g., Ref. [38].

In supersymmetry, box diagrams can change flavor unless specifically forbidden. Electroweaksymmetry-breaking schemes (for example, littlest-Higgs models with T-parity, technicolor, etc.) generically have flavor-changing interactions. Theories with extra dimensions (a recent example is Ref. [39]) can concentrate flavor violation in the top sector (a particular target for the International Linear Collider), and can possess Kaluza-Klein excitations at the TeV scale, accessible at the LHC.

\section{Dark matter scenarios}

Imagine a TeV-scale effective symmetry $\mathrm{SU}(3) \otimes \mathrm{SU}(2) \otimes \mathrm{U}(1) \otimes \mathrm{G}$, where the beyondStandard-Model (BSM) group G could be SUSY with R-parity, extra-dimensional excitations with Kaluza-Klein parity, little Higgs models with T-parity, technicolor, or some other group. One can classify the types of matter very generally as shown in Table [ [ [40]:

Table 2: Possible types of matter classified according to SM and BSM (G) transformation.

\begin{tabular}{cccc}
\hline \hline Type of matter & Std. Model & $\mathrm{G}$ & Example(s) \\
\hline Ordinary & Non-singlet & Singlet & Quarks, leptons \\
Mixed & Non-singlet & Non-singlet & Superpartners \\
Shadow & Singlet & Non-singlet & $E_{8}^{\prime}$ of $E_{8} \otimes E_{8}^{\prime}$ \\
\hline \hline
\end{tabular}

Ordinary matter could be singlets under $G$ even if its subconstituents were non-singlets (e.g., in composite-Higgs models). Loops could involve $G$-nonsinglets. Many dark matter scenarios involve mixed matter, such as superpartners or particles with odd KK- or T-parity. Flavor-changing loops can occur. Mixed-matter scenarios may be different if $\mathrm{G}$ is more general than a "parity." Shadow matter may not interact with ordinary matter at all except gravitationally.

\section{Hidden sector in loops}

Manifestations of a hidden sector interacting with ordinary matter are shown in Fig. 8. Mixed particles must have the same $\mathrm{SU}(3) \otimes \mathrm{SU}(2) \otimes \mathrm{U}(1)$ quantum numbers as the quarks to which they couple, but off-diagonal flavor couplings are allowed. Flavor-diagonal couplings still can affect 


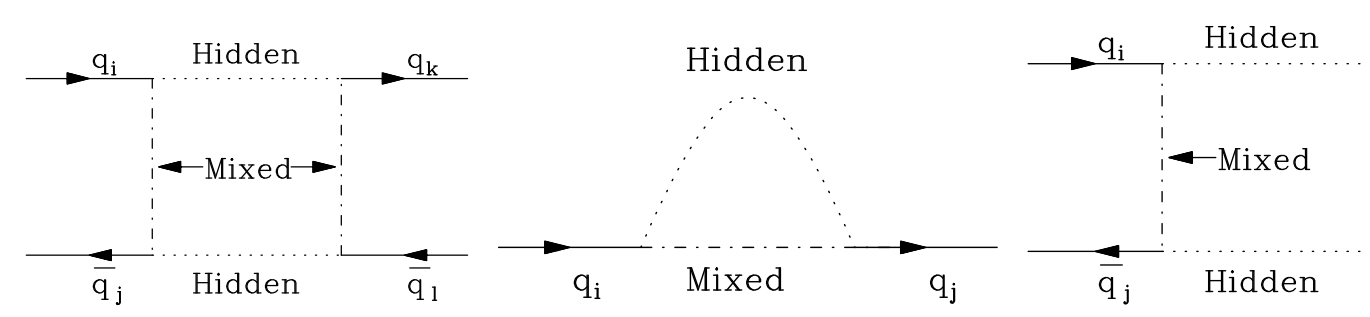

Figure 8: Hidden sector interacting with ordinary matter. Left: box diagram; center: penguin diagram; right: production of a pair of hidden-sector particles through exchange of a mixed state.

such quantities as the muon anomalous moment $a_{\mu}$, which has been shown particularly sensitive to new physics in some supersymmetry scenarios. For a coupling $\mathscr{O}(\alpha)$, the mass scale to explain the current $3 \sigma$ discrepancy in $a_{\mu}$ is $\sim 50 \mathrm{GeV}$.

In a recent paper, D. McKeen [41] suggests looking for light "hidden" states in quarkonium decay. For example, one can look for a light dark matter candidate $X$ in $\Upsilon(2 S) \rightarrow \gamma \chi_{b 0} \rightarrow \gamma X X$. This is one manifestation of the "WIMPless Dark Matter" scenario of Ref. [42].

\section{Some LHCb topics}

The LHCb experiment will provide a unique window to $B_{s}$ decays, through (e.g.) (1) Better $J / \psi \phi$ studies, with explicit time dependence plots. (2) $B_{s} \rightarrow J / \psi \eta$ : although $\mathscr{B}$ is less $(1 / 3$ of that for $J / \psi \phi$ ), no helicity analysis is needed. (3) $B_{s} \rightarrow J / \psi f_{0}$ : L. Zhang, in a poster at this Conference, estimates $\mathscr{B}\left(B_{s} \rightarrow J / \psi f_{0}\left(\rightarrow \pi^{+} \pi^{-}\right)\right) / \mathscr{B}\left(B_{s} \rightarrow J / \psi \phi\left(\rightarrow K^{+} K^{-}\right)\right)=(42 \pm 11) \%$. (4) A CP analysis involving $A\left(B_{s} \rightarrow D_{s}^{+} K^{-}\right) \sim V_{u b}^{*} V_{c s} ; A\left(\bar{B}_{s} \rightarrow D_{s}^{+} K^{-}\right) \sim V_{u s}^{*} V_{c b}$. (5) Comparisons of $\left(B, B_{s}\right) \rightarrow(\pi \pi, K \pi)$ [43, 44], yielding independent estimates of $\gamma$. (6) Many tests of flavor SU(3) by comparison with $B$ decays.

The hidden valley scenario [45] suggests an energy threshold (if we are lucky, the $\mathrm{TeV}$ scale) for the production of new matter; some may end up in new light (few GeV?) states. The LHCb Collaboration is aware of these possibilities, having discussed the examples of a $3 \mathrm{TeV} Z^{\prime}$, a 35 $\mathrm{GeV}$ "v-pion," and a SM Higgs devaying to a pair of v-pions [46].

Charm studies at LHCb will explore virgin territory because of the large production cross sections and small Standard Model CP violation. One will be able to probe loop and penguin diagrams involving the mixed and hidden sectors with unprecendented sensitivity.

\section{Looking forward}

Belle and the Fermilab Tevatron are still running; BaBar and CLEO are analyzing a rich trove of data. CLEO is capable of searching for light scalars or pseudoscalars in bottomonium decay, and the same is to be expected of the $B$ factories. In the nearest future we see results from BESIII [47] and from $\mathrm{LHCb}$ whenever the LHC begins operation, and some $b$ physics capabilities at ATLAS and CMS. Questions include many on the strange $B$ system, e.g., pinning down the mixing and/or the CP-violating phase in the $B_{s}-\bar{B}_{s}$ system. 
Other LHCb questions include: (a) flavor symmetry and departures from it in $B_{s}$ decays, to check schemes seeking to calculate strong-interaction properties (e.g., non-factorizable amplitudes); (b) effects of any new sector on loops and direct production of new particles.

The KEK-B/Belle upgrade will initially provide a data sample of $10 \mathrm{ab}^{-1}$ and eventually $>5$ times that; super-B more. A simple motivation for these machines is that anything studied previously with single- $B$ decays now can be studied with double-tagged events if tagging efficiency approaches $1 \%$. Going further in $e^{+} e^{-}$collisions, we will hope for an ILC to explore the Higgs, SUSY, and top sectors.

Finally, present experience with $B$ decays tells us that a rich program of understanding stronginteraction and nonperturbative effects will be needed to complement searches for rare processes in order to interpret apparent departures from the SM as genuine signs of new physics.

\section{Acknowledgments}

I would like to thank B. Bhattacharya, C.-W. Chiang, M. Gronau, M. Karliner, D. McKeen, B. Keren-Zur, H. Lipkin, D. Pirjol, A. Thalapillil, and my colleagues on the CLEO Collaboration for the opportunity to work together, and the organizers of FPCP2009 for a truly enjoyable and informative conference in a superb setting. This work was supported in part by the United States Department of Energy under Grant No. DE-FG02090ER40560.

\section{References}

[1] E. Komatsu et al. [WMAP Collaboration], Astrophys. J. Suppl. 180 (2009) 330.

[2] N. Cabibbo, Phys. Rev. Lett. 10 (1963) 531.

[3] M. Kobayashi and T. Maskawa, Prog. Theor. Phys. 49 (1973) 652.

[4] L. J. Hall, H. Murayama and N. Weiner, Phys. Rev. Lett. 84 (2000) 2572.

[5] J. L. Rosner, Phys. Rev. D 61 (2000) 097303.

[6] T. Aaltonen et al. [CDF Collaboration], Phys. Rev. D 76 (2007) 072006; C. Wolfe, Ph. D. thesis, University of Chicago (unpublished).

[7] A. Soni, this Conference; G. Hou, this Conference.

[8] LEP Electroweak Working Group, http:// lepewwg •web.cern.ch/LEPEWWG/

[9] M. E. Peskin and T. Takeuchi, Phys. Rev. Lett. 65 (1990) 964; Phys. Rev. D 46 (1990) 381.

[10] J. L. Rosner, Phys. Rev. D 65 (2002) 073026.

[11] B. Holdom et al., arXiv:0904.4698.

[12] L. Wolfenstein, Phys. Rev. Lett. 51 (1983) 1945.

[13] CKMfitter Collaboration, J. Charles et al., Eur. Phys. J. C 41 (2005) 1. Updated results may be found at http://ckmfitter.in2p3.fr/.

[14] UTfit Collaboration, M. Bona et al.. Updated results may be found at http://www . utfit.org/.

[15] Heavy Flavor Averaging Group (HFAG), E. Barberio et al.. Updated results may be found at http://www.slac.stanford.edu/xorg/hfag/. 
[16] E. Gamiz, C. T. H. Davies, G. P. Lepage, J. Shigemitsu and M. Wingate [HPQCD Collaboration], arXiv:0902.1815 [hep-lat].

[17] A. Abulencia et al. [CDF Collaboration], Phys. Rev. Lett. 97 (2006) 242003.

[18] R. A. Briere et al. [CLEO Collaboration], arXiv:0903.1681 [hep-ex], submitted to Phys. Rev. D.

[19] T. Aaltonen et al. [CDF Collaboration], Phys. Rev. Lett. 100 (2008) 161802.

[20] V. M. Abazov et al. [D0 Collaboration], Phys. Rev. Lett. 101 (2008) 241801.

[21] A. Chandra, Rencontres de Moriond EW 2009, La Thuile, Italy, March 7-14, 2009.

[22] A. Lenz and U. Nierste, JHEP 0706 (2007) 072.

[23] M. Gronau and J. L. Rosner, Phys. Lett. B 669 (2008) 321.

[24] A. J. Buras, Nucl. Phys. Proc. Suppl. 185 (2008) 157.

[25] M. Gronau and J. L. Rosner, Phys. Rev. D 59 (1999) 113002.

[26] C. W. Chiang, M. Gronau, J. L. Rosner and D. A. Suprun, Phys. Rev. D 70 (2004) 034020.

[27] H. n. Li and S. Mishima, arXiv:0901.1272 [hep-ph].

[28] A. B. Kaidalov and M. I. Vysotsky, Phys. Lett. B 652 (2007) 203.

[29] M. Gronau, Phys. Lett. B 627 (2005) 82.

[30] S. Baek, C. W. Chiang, M. Gronau, D. London and J. L. Rosner, Phys. Lett. B 678 (2009) 97.

[31] S. Dobbs et al. [CLEO Collaboration], Phys. Rev. D 79 (2009) 112008.

[32] C. Amsler al. [Particle Data Group], Phys. Lett. B 667 (2008) 1.

[33] J. Y. Ge [CLEO Collaboration], arXiv:0906.2138 [hep-ex], submitted to Phys. Rev. D.

[34] M. Gronau and J. L. Rosner, Phys. Rev. D 79 (2009) 074022.

[35] M. Gronau and J. L. Rosner, Phys. Rev. D 79 (2009) 074006.

[36] S. B. Athar et al. [CLEO Collaboration], Phys. Rev. Lett. 100 (2008) 181802.

[37] J. C. Pati and A. Salam, Phys. Rev. Lett. 31 (1973) 661; Phys. Rev. D 10 (1974) 275.

[38] P. Langacker and S. Uma Sankar, Phys. Rev. D 40 (1989) 1569.

[39] A. L. Fitzpatrick, L. Randall and G. Perez, Phys. Rev. Lett. 100 (2008) 171604.

[40] J. L. Rosner, "Dark matter in many forms," arXiv:astro-ph/0509196, presented at 2005 ALCPG \& ILC Workshops, Snowmass, Colorado, August 14-27, 2005, published in the Proceedings [ECONF C0508141, ALCPG0106, (2005)].

[41] D. McKeen, Phys. Rev. D 79 (2009) 114001.

[42] J. L. Feng and J. Kumar, Phys. Rev. Lett. 101 (2008) 231301.

[43] R. Fleischer, Phys. Lett. B 459 (1999) 306.

[44] M. Gronau and J. L. Rosner, Phys. Lett. B 482 (2000) 71.

[45] M. J. Strassler and K. M. Zurek, Phys. Lett. B 651 (2007) 374; T. Han, Z. Si, K. M. Zurek and M. J. Strassler, JHEP 0807 (2008) 008.

[46] M. Kuharczyk and S. Stone, "Status of Hidden Valley in LHCb," Exotica Workshop, LHCb week, May 26, 2009.

[47] R. Briere, this Conference. 